\title{
Using Clinical Audits in Complex Allied Health Interventions: Challenges, Barriers, and Application - Our Experience Applied in Service Delivery for Children with Autism Spectrum Disorder
}

\author{
Weeks, S.; Boshoff, K.; Milanese, S.; Stewart, H. \& Grimmer, K. International Centre for \\ Allied Health Evidence, Allied Health and Human Performance, University of South Australia
}

DOI: 10.21913/TAHS.v3i1.1619

\begin{abstract}
This paper discusses the use of retrospective clinical audits in complex Allied Health interventions, specifically based on our experience of designing and trialling a clinical audit in one service delivery area of Occupational Therapy (i.e., Occupational Therapy for sensory needs of children with autism spectrum disorder). Covered in our discussion is the application considerations of conducting an audit as external auditors to service organisations, however, lessons learnt can be applied to other Allied Health service delivery contexts as well. We encourage Allied Health professionals and researchers to utilise clinical audits as the foundation for decision-making and especially for further research in areas with limited efficacy- and effectiveness-based research.
\end{abstract}

\section{Keywords}

Clinical audit, autism spectrum disorder, sensory, complex interventions 


\section{Introduction: A call for the use of clinical audits in Allied Health}

As part of doctoral research, we proposed as external auditors to conduct a retrospective clinical record audit to describe community Occupational Therapy for sensory difficulties in children on the autism spectrum. The aim of this viewpoint is to discuss a range of topic specific and procedural barriers we encountered as external auditors with community Occupational Therapy clinical records. We also present the values and benefits identified by an expert panel of nine senior occupational therapists on developing audit criteria and a process for self-auditing their clinical records, in response to these challenges. The panel were identified from a sampling frame generated for this research (Weeks \& Atlas, 2015) and had a minimum of 10 years-experience as occupational therapists (mean 24 years registered; mean 20 years in paediatric Occupational Therapy; mean 17 years working with autism). The panel were employed across a range of public, private, non-government, and special school sectors in current and previous roles (four employed across three sectors, four across two sectors, and one in one sector).

For almost a century, clinical research in medicine and health has been guided by retrospective systematic investigation of clinical records (Gearing et al., 2006). Clinical audit is defined as:

A quality improvement process that seeks to improve patient care and outcomes through systematic review of care against explicit criteria and the implementation of change. Aspects of the structure, processes, and outcomes of care are selected and systematically evaluated against explicit criteria. Where indicated, changes are implemented at an individual, team, or service level and further monitoring is used to confirm improvement in healthcare delivery (National Institute for Clinical Excellence, 2002, p. 1). 
Structure aspects in clinical audits investigate the physical attributes or resources required for healthcare service delivery, including organisational structures, staff numbers and skills, medical supplies and equipment, and built environment. Process aspects investigate actions and decisions made by health services or practitioners in consultation with clients, including assessments, interventions, prescriptions, education, and communication. Outcome aspects investigate changes in client health status following intervention, such as physical, cognitive, or behavioural changes, and the client's level of knowledge and satisfaction (National Institute for Clinical Excellence, 2002). Clinical audits have historically been conducted for clinical research, evaluation of inpatient, outpatient and community care, adherence to clinical guideline standards, epidemiological studies, quality assessment, and professional education and development (Gearing et al., 2006; Sarkar \& Seshadri, 2014). Our proposed study objective aligned with the clinical audit 'processes' aspect of service delivery. We intended to use the results of a full-scale audit to prioritise and design intervention effectiveness trials, with real-world applicability to community Occupational Therapy organisations and children on the autism spectrum, to progress the evidence-base in this field. Our rationale for effectiveness research was two-fold. Firstly, Lord et al., (2005) advise to move directly to effectiveness trials if interventions are well established in community practice, therefore by-passing efficacy trials (i.e., how an intervention works under tightly controlled and ideal settings). Occupational Therapy interventions for sensory difficulties in autism are well established in Australian community practice (Ashburner et al., 2014; Kadar et al., 2012). Secondly, most universities have insufficient funding to conduct informative intervention effectiveness research in complex areas, whilst most practitioners have insufficient knowledge of complex intervention research design or and limited time for research supplementary to daily practice and reporting requirements (Lord et al., 2005). Pragmatically combining academia and clinician resources to incorporate multi-site designs 
affords increased sample sizes, pooling of data (Lord et al., 2005) and representation of what is occurring in the wider community.

Although sensory difficulties in children on the autism spectrum is used to facilitate this discussion, we believe that these examples could inform other researchers and clinicians undertaking clinical audits in practice areas with complex diagnoses and complex interventions. Through our experience, the benefits of the use of clinical audits are evident and we call on Allied Health professionals and researchers to heighten their use of clinical audits in practice, in order to lay the foundation for decision-making and further research, especially in areas with limited effectiveness-based research.

\section{What was the preparatory process for audit?}

It is important to construct a data extraction instrument with a logical order, and if possible, parallels the flow of documentation in the clinical records (Gearing et al., 2006). A well-designed audit instrument is guided by research aims and the coding plan considers which variables will be extracted and how they will be coded a priori to data extraction (Sarkar \& Seshadri, 2014). Audit variables are then operationalised by transforming scientific concepts into definable terms and categories (Vassar \& Holzmann, 2013). This occurred through two iterative stages. Audit variables that were straight forward, universally accepted, or well-understood in the community were identified and defined through a literature review and research team discussions (Gearing et al., 2006; Vassar \& Holzmann, 2013). We developed the overarching structure of the audit instrument by using two reporting standards for intervention effectiveness studies (Gutman, 2010; Moher et al., 2010) and a critical appraisal instrument for randomised controlled trials (Maher et al., 2003). Moreover, child and family demographics, sensory classification and assessment, and outcomes sections were developed using this process. We concurrently conducted a scoping review (Weeks, 2019) on Occupational Therapy interventions for sensory difficulties in children on the autism 
spectrum to determine how other researchers operationalised key audit variables because there was less clarity about terminology and description which required in-depth consideration (Vassar \& Holzmann, 2013).

A pilot audit on a small sample of clinical records should then be conducted to determine if the operationalised audit items can be populated from clinical documentation (Gearing et al., 2006; Vassar \& Holzmann, 2013) by checking for availability, completeness, accuracy (Dixon, 2013; Dixon \& Pearce, 2010) and missing data (Gearing et al., 2006; Vassar \& Holzmann, 2013). This process also evaluates the reliability of the instrument and external auditors before proceeding to a full-scale audit (Gearing et al., 2006). We therefore conducted a retrospective pilot audit of de-identified clinical records from a private paediatric Occupational Therapy organisation that employed multiple occupational therapists and administered a range of interventions identified in the doctoral scoping review.

\section{What did we find?}

Information required for retrospective audits may not be documented consistently in clinical records (Jansen et al., 2005) because their initial purpose was to directly support client care (Mann \& Williams, 2003). Illegibility and difficulty interpreting documentation are common in clinical records using free-text format, which can cause invalid and unreliable data (Worster \& Haines, 2004). Our pilot audit found over 20 percent of missing data due to mutually exclusive categories: a) confirmation bias by external auditors and b) information not documented. The parameter for the "confirm bias" criteria was that information was documented in clinical records that potentially answered the audit item, however, the risk of an external auditor misinterpreting the documentation was too high to accurately extract data. Conversely, the parameters for the "information not documented" criteria needed to contain both lack of documentation on the audit item and no evidence the item was asked for or considered by the occupational therapist. Whilst there is no universally agreed to rate of 
acceptable missing data, it is indicated that audit items over 10\% (Worster \& Haines, 2004), and over 20\% for clinical parameters (Jansen et al., 2005), be removed from larger audits because they can elicit a non-response bias of results (Gearing et al., 2006; Worster \& Haines, 2004). However, most of our audit items with missing data could not be removed because they were paramount to answering our research question.

The clinical records piloted were densely populated with information that was meaningful to the occupational therapists and predominantly able to be understood by colleagues with knowledge of their organisation's internal practice and professional reasoning. The concern was the amount of information unable to be accurately extracted by an external auditor. If one organisation with a structured assessment process and intervention practices varied to this degree in their practitioner's clinical documentation, questions were raised about the potential variability that could be found across multiple organisations. Likewise, the feasibility of external auditors accurately extracting data was also of concern in a full-scale audit with our current understanding of clinical documentation.

In retrospective audits, a data validation process is recommended during data analysis to assist with interpretating data outliers or unclear results (Dixon \& Pearce, 2010). We had initially planned to engage the expert panel for data validation upon the completion of the proposed full-scale audit, however, brought this process forward due to the unanticipated results of the pilot audit. The expert panel were convened to inform how these issues could be addressed, if an audit of clinical practice by an external auditor was a valid and feasible approach for our research, and if an audit would have value to community Occupational Therapy organisations. The expert panel conferred that the similar issues would exist across public, private, and non-governments organisation clinical records and expanded on our understanding. 


\section{Sensory specific contributions to missing data}

\section{Challenges interpreting sensory difficulties in clinical records}

Our audit aimed to identify the classifications community occupational therapists were using to confirm sensory phenotypes and how they described them. This information would be used to evaluate overlap and divergence in sensory classifications (i.e., are the same terms used to classify different disorders, or conversely, different terms used to classify the same disorder), inform inclusion and exclusion criteria for sensory difficulties in an intervention trial and determine the feasibility of including homogenous groups of participants. Shorter term objectives could inform future studies to obtain consensus or best practice research for sensory classification (e.g., Delphi study).

The fifth edition of the Diagnostic and Statistical Manual of Mental Disorders (DSM5) formally recognised sensory differences as a core feature of autism spectrum disorder (ASD) (American Psychiatric Association, 2013). Hyper- or hypo-reactivity to sensory input or unusual interest in sensory aspects of the environment became additional criterion for restricted and repetitive patterns of behaviour. These are described in DSM-5 as responding negatively to specific sounds or textures, the need to excessively smell or touch objects, in having a visual attraction to lights and movement, and pain or temperature indifferences (American Psychiatric Association, 2013). Schaaf and Lane (2015) identified an extensive range of sensory classifications, with respective descriptions, reported in peer-reviewed literature for individuals on the autism spectrum. In an effort to lessen confusion about the variability of classifications used in the literature, the authors allocated sensory classifications with synonymous descriptions to either sensory hyper-reactivity, sensory hypo-reactivity, or unusual sensory interest categories. Additional sensory classifications were also identified that were not consistent with DSM-5 sensory criterion, such as sensory perception and sensory integration/multi-sensory integration differences. This highlighted that the 
taxonomies for sensory differences reported in the literature are broader than those included in the DSM-5 (Schaaf \& Lane, 2015).

For our specific service delivery context, confirmation bias and lack of documentation on sensory classifications had the potential to either over- or under-represent the range and prevalence being identified by community occupational therapists. The primary barrier in the pilot audit was an unanticipated low occurrence of sensory classifications documented. This raised an important question about why sensory difficulties reported in peer-reviewed literature were being described in clinical records, but not classified using phenotypes. The panel agreed with the overlap of sensory classifications and descriptions for hyper- and hyporeactivity, which had a higher potential for availability and accuracy in clinical records. The panel were less confident that classifications for unusual sensory interests would be documented consistently.

The main challenge identified by the panel was how to decide if the child's behaviours or interests were in fact an unusual response to sensory input, with grey areas around what actions, contexts, intensity, and duration qualify the child's response to sensory input as unusual. On the one hand, sensory behaviours such as moving away from pain if it hurts, or the perception it is going to hurt, were not considered unusual. On the other hand, sensory interests such as children over three years of age placing items such as rocks, batteries, dog faeces, or grass in their mouths were clearly unusual. The challenges reported were what might seem unusual in one person's home might not be in another's; what might be unusual in one context might not be in another (e.g., jumping when playing compared to jumping when the child is required to maintain regulation and sit still); and strategies that children on the autism spectrum use to self-regulate, such as hand flapping, might also be used by neurotypical children. These issues were more likely to be described in documentation without providing a sensory classification. Moreover, the panel reported 
practitioners were hesitant to provide formal labels for sensory classifications too soon, as caregivers often reported fluctuations in their child's sensory responses, either in severity or between sensory symptoms and typical responses, over three- to four-week periods.

Intuitional factors also influenced whether sensory classifications were documented. A decrease in the use of sensory classifications had been influenced by funding restrictions for sensory-based interventions under the former Helping Children with Autism (HCWA) package. It was reported that some case managers would misinterpret information in Occupational Therapy reports and any report including a sensory classification (e.g., sensory input, sensory regulation, sensory modulation) would have interventions automatically declined, even if the interventions were not sensory based. The term 'self-regulation problems' was acceptable to HCWA case managers, and occupational therapists frequently placed sensory difficulties under this label. Likewise, this carried over to the National Disability Insurance Scheme (NDIS) with goals provided by case managers related to the child's functional and behavioural challenges. Similarly, NDIS funding planners used 'emotional-regulation' as goals in their plans, which provided an entry point for occupational therapists to recommend intervention strategies for sensory difficulties. An example provided was documenting and reporting 'assisting with self-regulation and ability to manage emotional-regulation' in lieu of using sensory modulation as a classification. A sensory difference may have underpinned part, or all of the difference in behaviour, however not carried over to formal reports. The lack of documentation or confirmation bias of sensory phenotypes would impact on eligibility in an effectiveness trial.

\section{Challenges interpreting sensory and non-sensory assessments in clinical records}

The characterisation of sensory difficulties is essential to plan and tailor interventions and outcomes measures to individuals with ASD (Schaaf \& Lane, 2015; Watling \& Hauer, 2015). Recommendations for an initial assessment of sensory difficulties vary from 
administering at least one published standardised assessment (Watling \& Hauer, 2015) to administering multiple standardised assessments to assess for both sensory reactivity and sensory perception and integration difficulties (Schaaf \& Lane, 2015). The evaluation process should also involve structured observations and informal assessments to supplement and interpret the results of the standardised assessment (i.e., to support or contrast findings), with regards to the child's sensory preferences or difficulties and within the contexts of different daily activities and environments (Tomchek \& Koenig, 2016).

Our pilot audit was able to extract data on sensory standardised assessments due to the organisations' structured assessment practices. However, the panel advised that some caregivers may have difficulty completing a sensory-based standardised assessment because of the complex language around sensory features in autism. Some of these assessments are proxy reports completed by caregivers in questionnaire format. Caregivers who may lack capacity (e.g., have an intellectual impairment), have cultural differences in what is considered typical behaviour, or have limited proficiency in spoken English can misinterpret assessments. In these circumstances, occupational therapists may use the questionnaire as an interview guide to elicit more accurate information and prevent having to repeat the assessment (e.g., results of the caregiver assessment might conflict with observations by the practitioner). Altering how standardised assessments are administered threatens the validity and reliably of the assessment, which are important factors for intervention effectiveness trials. The expert panel advised that alterations to delivery of assessments is not usually documented. This was also extended to administering sub-tests or sections relevant to the child's concerns, rather than implementing the full version.

Occupational therapists also administer assessments that are not sensory based to either help confirm the presence of a sensory disorder or to identify the impacts sensory difficulties have on other occupational, adaptive, functional, or behavioural issues. Whilst we 
could capture at least the names of sensory-based standardised assessments in the pilot audit and align this up with a sensory classification, we were advised that there would be higher degree of confirmation bias for non-sensory standardised or informal assessments to identify the presence of a sensory difficulty. These assessments could be valuable outcome measures in an intervention trial.

\section{Challenges interpreting interventions and outcomes in clinical records}

Guidelines for the standard of documentation by occupational therapists recommend maintaining clear and concise clinical records to enable quality of care, optimal service delivery, continuity of care, and detailed information for colleagues. Appropriate documentation should be legible, accurate, factual, and report relevant details in a form that can be understood by other health practitioners (Occupational Therapy Board of Australia, 2014).

Watling and Hauer (2015) proposed that conflicting and confusing intervention effectiveness results could be related to the use of inaccurate and misinformed terminology for sensory interventions and advise practitioners of their responsibility to use accurate and precise terminology in their documentation. This rationale underpinned our scoping review that identified key intervention variables to code in the audit. A range of sensory and nonsensory interventions for sensory difficulties, implemented by occupational therapists, were identified and terminology and descriptions were mapped. Moreover, additional variables were identified that could help interpret the what the intervention was and inform coding for an intervention effectiveness trial. These included theoretical underpinnings (e.g., impairment or performance approach), service delivery models, who implements intervention, whether the intervention was child- or adult-directed, setting/s, equipment used, and treatment intensity (i.e., dosage). 
We were able to capture more data on interventions at a programs, techniques, and strategies level in the pilot audit, which the panel confirmed would be the data we were more likely to see documented across organisations. The challenges we experienced in the pilot audit were difficulties interpreting the majority of diagrams used (>80\%) in clinical intervention sessions for activities and equipment, and no documentation or legends were provided to explain diagrams. There was also inconsistency in diagrams documented between occupational therapists (i.e., appeared to use different diagrams for similar activities). Similarly, for targeted outcomes of sessions, single words are often noted in session notes (sensory, touch, coordination) or with shorthand (e.g., $\downarrow$ balance; $\downarrow$ sensitivity to touch). It was unclear if these were a sensory presentation on the day, an aim of the session, or a posttest following the intervention session. To follow on from the sensory assessment issue of being able to confirm behaviours linked to sensory, an example target outcome was noted ( $\uparrow$ arousal). It was unclear if arousal was affected by a sensory or non-sensory difficulty, which senses or combination of senses were causing the problems with arousal; and whether the child was over-aroused, and the aim was to decrease arousal, or vice-versa.

It was also unclear what senses and sensory difficulties were targeted per intervention session (senses targeted documented in $<20 \%$ of records) and the proportion of intervention time spent (minutes or \%) per sense/sensory difficulty was not documented. There was no documentation on theoretical underpinnings, service delivery models and whether intervention was child- or adult-directed. The panel were less confident we would see evidence of these items documented across organisations.

Moreover, Reynolds et al. (2017) conceptualised a multifaceted approach for intervention with individuals who have sensory difficulties. This framework categorised intervention approaches into three overarching domains, including a) environmental supports and adaptations to enhance success; b) child-focussed interventions, including Ayres Sensory 
Integration ${ }^{\circledR}$, sensory-based interventions, behavioural, cognitive, biomechanical, and practice and developmental skill building approaches; and c) caregiver-focussed interventions, including coaching, parent- and teacher-mediated interventions, and education (Reynolds et al., 2017). It was unclear in the pilot audit clinic-based intervention sessions if co-interventions were being implemented and this would need to be confirmed by the occupational therapist. For instance, the panel advised that therapists may be using language consistent with a cognitive or behavioural approach during an Ayres Sensory Integration ${ }^{\circledR}$ or sensory-based intervention, but not document it.

Lastly, another barrier to capturing intervention data would stem from the sensory classification barriers mentioned above. Without being able to accurately identify sensory difficulties in clinical records, we potentially miss important interventions.

\section{Procedural challenges in locating and extracting data}

\section{Documentation not in Occupational Therapy clinical records}

In response to an example of intervention that was recommended but no evidence that it had been received, the panel advised some documentation will be kept in locations other than Occupational Therapy clinical records within the same site. For example, compensatory sensory equipment (e.g., noise cancelling headphones) purchased for use in home and community settings may be stored within various folders or electronic practice management software according to funding scheme and accessed by administration staff. Therapeutic session notes in 'blocks' of therapy (i.e., multiple occasions of service within an episode of care) may still be on the occupational therapist's desk or in a working folder until the current episode of care concludes. Likewise, consultation with other health practitioners, teachers, and caregivers may have taken place in emails or by phone and not transferred to clinical records. 
The panel also advised that documentation may also be stored in other practitioner's electronic files, such as autism diagnostic reports in multidisciplinary organisations, which the occupational therapist has access to, but is not the custodian of. Moreover, organisations that provide mobile services or have multiple sites advised that some clinical information can be stored at a central site and would be difficult for an external auditor to access if the organisation's central ethics committee was responsible for their release.

\section{Time required to identify and de-identify eligible clinical records}

External auditors are typically permitted to read clinical documentation containing identifiable information, however, the data extracted and reported must be de-identified (Vassar \& Holzmann, 2013). Conversely, ethics approval for our proposed clinical audit required participating organisations to de-identify their clinical records prior to handing them over to the external auditor for data extraction. The ethics committee's rationale was to add another layer of protection to vulnerable children and their families

Because external auditors were prevented from scanning clinical records with identifiable information, the time taken for occupational therapists to identify eligible clinical records themselves presented a perceived barrier by the panel for recruitment into the audit. Organisations with electronic practice management software advised they could not perform key word searches to identify clinical records of children with a formal diagnosis of ASD to mitigate this issue. Instead, clinical records would have to be hand searched the same as organisations who keep paper records in manual lateral filing systems. As most paediatric occupational therapists work with children who have a range of clinical conditions, children eligible for this research may not be current clients and the occupational therapist may have to spend time searching files for eligible records that otherwise could be allocated to research. Moreover, one of the benefits of an external auditor extracting data from clinical records is to reduce the time commitment of participant organisations and occupational therapists, who 
have already contributed to the research by providing service delivery and through maintaining their clinical records. Our pilot audit identified a range of approximately 50 to 150 identifying entries that would need removal per child clinical records before being released to the research team. The panel were concerned that the time spent identifying eligible records and then de-identifying entries would use the time they had put aside for research and void the benefit of an external auditor.

\section{What were the values and benefits of an audit by the expert panel?}

To mitigate confirmation bias issues described above, the expert panel thought acceptable completion rates might be achievable if the external auditor had regular access to occupational therapists for confirmation of entries. By the time entries were confirmed, records were identified and deidentified, and follow up information about missing data was sourced from other records, practitioners, or central ethics committees, a self-audit appeared to be more feasible.

One expert panel member shared their experience with self-auditing their practitioners' clinical records for weighted vest recommendations and client outcomes for children on the autism spectrum. This was undertaken because of inconsistency with NDIS funding approvals and the paucity of research evidence on weighted vests. The expert panel member stated a validated set of audit criteria would have increased their confidence in the process, as they were uncertain if they were asking the correct questions. If a validated set of audit criteria existed, they would implement more self-audits to measure the safety and effectiveness of their interventions.

Continuing professional development (CPD) activities contribute to the knowledge, expertise, and competence of occupational therapists (Occupational Therapy Australia, 2016). Internal audits are well established as an activity for CPD in medical professions to maintain an active Fellowship (Birch et al., 2005) and self-appraisal of one's own practice is 
considered a key aspect of learning (Gagliardi et al., 2011). Similarly, registered occupational therapists are mandated to complete CPD per annum, part of which can be obtained by participating in a clinical audit or review of records (Occupational Therapy Board of Australia, 2019).

The expert panel identified the following benefits for a self-audit process and criteria on this service delivery topic:

- Validating a foundational set of audit criteria to describe service delivery that could be implemented by academics for research and practitioners for internal organisation audits

- Identifying aspects of current clinical documentation that could be improved to describe sensory classification, assessment, and intervention adequately

- Improving client intake forms and assessment processes

- Prioritising and designing intervention effectiveness research with real-world applicability to children on the autism spectrum and community-based occupational therapists, and within the context of current funding schemes

- Providing feedback to case managers and funding schemes about inconsistencies in intervention approvals for sensory difficulties in children on the autism spectrum

- Potentially identifying novel interventions in this field (longer-term and larger audit)

- A training tool for new graduates and early career occupational therapists

- Informing standardisation of how Occupational Therapy for sensory difficulties in autism is taught to students

- Developing a manual of audit procedures (e.g., data dictionary) which trains occupational therapists to a level where they consider intervention effectiveness as part of their practice and how service delivery should be documented in a more consistent manner 


\section{In summary}

Recommendations for future research on sensory interventions for individuals on the autism spectrum include several key areas that align with the aims of our proposed full-scale audit. These include engaging community-based occupational therapists in research activities to identify new research questions arising from clinical issues; conducting research to tailor interventions to specific sensory phenotypes; conducting studies on optimal intervention intensity for specific sensory phenotypes; and conducting this research through multi-site studies (Schaaf et al., 2015). Whilst these recommendations were for Ayres Sensory Integration ${ }^{\circledR}$, they are equally relevant for each of the multifaceted approaches proposed by (Reynolds et al., 2017) and could have relevance to most complex interventions.

The safety and effectiveness of Occupational Therapy service delivery is the responsibility of all occupational therapists (American Occupational Therapy Association, 2020). Doucet, Woodson et al. (2014) encourage occupational therapists to be courageous with their quest to expand the profession's knowledgebase by incorporating research into clinical practice, and in partnership with researchers, develop meaningful clinical practice research questions. Careful attention should be directed at who will benefit from specific interventions due to the heterogeneity of ASD, as interventions are not always appropriate for all individuals on the autism spectrum (Bagatell \& Mason, 2015).

This paper discusses the use of retrospective clinical audits in complex Allied Health interventions, specifically based on our experience of designing and trialling a clinical audit in one service delivery area of Occupational Therapy (i.e., Occupational Therapy for children with Autism Spectrum Disorder). Covered in our discussion is the application considerations of conducting an audit as external auditors to service organisations, however, lessons learnt can be applied to other Allied Health service delivery contexts as well. We encourage Allied Health professionals and researchers to utilise clinical audits as the foundation for decision- 
making and especially for further research in areas with limited efficacy- and effectivenessbased research.

\section{Key Points}

- Clinical audits aim to improve patient care and healthcare service delivery through systematic evaluation of clinical records and changes can be implemented at an individual, team, or service levels

- External auditors conducting retrospective clinical audits in Allied Health areas with complex diagnoses and complex interventions may encounter missing data through confirmation bias, information not documented, and procedural barriers using community clinical records because their initial purpose was to directly support client care and not research

- Clinical record audits have many benefits for informing research and education of Allied Health practitioners and participation in self-audits can be used for continuing professional development hours per annum

- Allied Health professionals and researchers are encouraged to combine resources and utilise clinical audits as foundation of decision-making and especially for further research in areas with limited efficacy- and effectiveness-based research

\section{Conflicts of Interest}

There are no conflicts of interest to declare for this publication.

\section{Funding Statement}

The authors received no specific funding for this study.

\section{Ethics Approval}

Ethical approval for the pilot audit was obtained from the University of South Australia’s Human Research Ethics Committee (protocol number: 0000031942). 


\section{References}

American Occupational Therapy Association. (2020). Occupational therapy practice framework: domain \& process (4th edition). American Journal of occupational therapy, 74(Supplement. 2). https://doi.org/10.5014/ajot.2020.74S2001

American Psychiatric Association. (2013). Diagnostic and statistical manual of mental disorders (5th ed.). American Psychiatric Association.

Ashburner, J., Rodger, S., Ziviani, J., \& Jones, J. (2014). Occupational therapy services for people with autism spectrum disorders: current state of play, use of evidence and future learning priorities. Australian Occupational Therapy Journal, 61(2), 110-120. https://doi.org/http://dx.doi.org/10.1111/1440-1630.12083

Bagatell, N., \& Mason, A. E. (2015). Looking backward, thinking forward: occupational therapy and autism spectrum disorders. OTJR: Occupation Participation and Health, 35(1), 34-41. https://doi.org/10.1177/1539449214557795

Birch, D. W., Goldsmith, C. H., Tandan, V., \& for the Evidence-Based Surgery Working, G. (2005). Users' guide to the surgical literature: self-audit and practice appraisal for surgeons. Canadian Journal of Surgery, 48(1), 57-62. http://www.ncbi.nlm.nih.gov/pmc/articles/PMC3211577/

Dixon, N. (2013). Proposed standards for the design and conduct of a national clinical audit or quality improvement study. International Journal for Quality in Health Care, 25(4), 357-365. https://doi.org/10.1093/intqhc/mzt037

Dixon, N., \& Pearce, M. (2010). Guide to ensuring data quality in clinical audits. http://hqip.org.uk/assets/LQIT-uploads/Guidance-0112/HQIP-CA-PD-028-Guide-toEnsuring-Data-Quality-in-Clinical-Audits-251111.2.pdf 
Doucet, B. M., Woodson, A., \& Watford, M. (2014). Moving toward 2017: progress in rehabilitation intervention effectiveness research. American Journal of occupational therapy, 68(4), e124-e148. https://doi.org/10.5014/ajot.2014.011874

Gagliardi, A. R., Brouwers, M. C., Finelli, A., Campbell, C. E., Marlow, B. A., \& Silver, I. L. (2011). Physician self-audit: a scoping review. Journal of Continuing Education in the Health Professions, 31(4), 258-264. https://doi.org/doi:10.1002/chp.20138

Gearing, R. E., Mian, I. A., Barber, J., \& Ickowicz, A. (2006). A methodology for conducting retrospective chart review research in child and adolescent psychiatry Journal of the Canadian Academy of Child and Adolescent Psychiatry, 15(3), 126-134. http://www.ncbi.nlm.nih.gov/pmc/articles/PMC2277255/

Gutman, S. A. (2010). Reporting standards for intervention effectiveness studies. American Journal of occupational therapy, 64(4), 523-527. https://doi.org/10.5014/ajot.2010.09644

Jansen, A. C. M., van Aalst-Cohen, E. S., Hutten, B. A., Büller, H. R., Kastelein, J. J. P., \& Prins, M. H. (2005). Guidelines were developed for data collection from medical records for use in retrospective analyses. Journal of Clinical Epidemiology, 58(3), 269-274. https://doi.org/10.1016/j.jclinepi.2004.07.006

Kadar, M., McDonald, R., \& Lentin, P. (2012). Evidence-based practice in occupational therapy services for children with autism spectrum disorders in Victoria, Australia. Australian Occupational Therapy Journal, 59(4), 284-293. https://doi.org/http://dx.doi.org/10.1111/j.1440-1630.2012.01015.x

Lord, C., Wagner, A., Rogers, S., Szatmari, P., Aman, M., Charman, T., Dawson, G., Durand, V. M., Grossman, L., Guthrie, D., Harris, S., Kasari, C., Marcus, L., Murphy, S., Odom, S., Pickles, A., Scahill, L., Shaw, E., Siegel, B., Sigman, M., Stone, W., Smith, T., \& Yoder, P. (2005). Challenges in evaluating psychosocial interventions 
for autistic spectrum disorders [journal article]. Journal of autism and developmental disorders, 35(6), 695-708. https://doi.org/10.1007/s10803-005-0017-6

Maher, C. G., Sherrington, C., Herbert, R. D., Moseley, A. M., \& Elkins, M. (2003).

Reliability of the PEDro scale for rating quality of randomized controlled trials Physical Therapy, 83(8), 713-721. https://doi.org/10.1093/ptj/83.8.713

Mann, R., \& Williams, J. (2003). Standards in medical record keeping. Clinical Medicine, 3(4), 329-332. https://doi.org/10.7861/clinmedicine.3-4-329

Moher, D., Hopewell, S., Schulz, K. F., Montori, V., Gøtzsche, P. C., Devereaux, P. J., Elbourne, D., Egger, M., \& Altman, D. G. (2010). CONSORT 2010 Explanation and Elaboration: updated guidelines for reporting parallel group randomised trials. $B M J$, 340, c869. https://doi.org/10.1136/bmj.c869

National Institute for Clinical Excellence. (2002). Principles for best practice in clinical audit. Radcliffe Medical Press.

Occupational Therapy Australia. (2016). Occupational therapy guide to good practice: working with children.

Occupational Therapy Board of Australia. (2014). Code of conduct. Occupational Therapy Board of Australia, . Retrieved 10 September 2015 from http://www.occupationaltherapyboard.gov.au/Codes-Guidelines/Code-ofconduct.aspx

Occupational Therapy Board of Australia. (2019). Guidelines: continuing professional development. Occupational Therapy Board of Australia, . file://C:/Users/weekssl/Downloads/Occupatoinal-Therapy-Board---Registrationstandard---Continuing-professional-development.PDF

Reynolds, S., Glennon, T. J., Ausderau, K., Bendixen, R. M., Kuhaneck, H. M., Pfeiffer, B., Watling, R., Wilkinson, K., \& Bodison, S. C. (2017). Using a multifaceted approach 
to working with children who have differences in sensory processing and integration [Article]. American Journal of occupational therapy, 71(2), 1-10, Article 7102360010. https://doi.org/10.5014/ajot.2017.019281

Sarkar, S., \& Seshadri, D. (2014). Conducting record review studies in clinical practice. Journal of Clinical and Diagnostic Research 8(9), JG01-JG04. https://doi.org/10.7860/JCDR/2014/8301.4806

Schaaf, R., \& Lane, A. (2015). Toward a best-practice protocol for assessment of sensory features in ASD [Article]. Journal of Autism \& Developmental Disorders, 45(5), 1380-1395. https://doi.org/10.1007/s10803-014-2299-z

Schaaf, R. C., Schoen, S. A., May-Benson, T. A., Parham, L. D., Lane, S. J., Roley, S. S., \& Mailloux, Z. (2015). State of the science: a road map for research in sensory integration [Editorial Material]. American Journal of occupational therapy, 69(6), 7, Article 6906360010. https://doi.org/10.5014/ajot.2015.019539

Tomchek, S., \& Koenig, K. (2016). Occupational therapy practice guidelines for individuals with autism spectrum disorder: the AOTA practice guidelines series.

Vassar, M., \& Holzmann, M. (2013). The retrospective chart review: important methodological considerations. Journal of Educational Evaluation for Health Professions, 10, 12. https://doi.org/10.3352/jeehp.2013.10.12

Watling, R., \& Hauer, S. (2015). Effectiveness of Ayres Sensory Integration and sensorybased interventions for people with autism spectrum disorder: a systematic review. American Journal of occupational therapy, 69(5), 1-12. https://doi.org/https://dx.doi.org/10.5014/ajot.2015.018051

Weeks, S. (2019). Developing foundations for robust occupational-therapy-based intervention studies to address the sensory needs of children with Autism Spectrum 
Disorder in Australia Thesis ( $\mathrm{PhD}$ (Health Sciences))--University of South Australia, 2019.].

Weeks, S., \& Atlas, A. (2015). Clinical audit for occupational therapy intervention for children with autism spectrum disorder: sampling steps and sample size calculation. BMC Research Notes, 8(1), 282. http://www.biomedcentral.com/1756-0500/8/282

Worster, A., \& Haines, T. (2004). Advanced statistics: understanding medical record review (MRR) studies. Academic Emergency Medicine, 11(2), 187-192.

https://doi.org/doi:10.1111/j.1553-2712.2004.tb01433.x 